Esta revista forma parte del acervo de la Biblioteca Jurídica Virtual del Instituto de Investigaciones Jurídicas de la UNAM www.juridicas.unam.mx

http://biblio.juridicas.unam.mx

\title{
LA INTRODUCCIÓN DE LA TEORÍA DE LOS SISTEMAS DE NIKLAS LUHMANN EN LA FILOSOFÍA JURÍDICA Y SOCIAL ARGENTINA
}

\author{
THE ADOPTION OF NIKLAS LUHMANN'S SYSTEMS THEORY \\ IN ARGENTINE LEGAL AND SOCIAL PHILOSOPHY
}

Jorge E. DOUGLAS PRICE*

Resumen:

Una teoría puede ser una explicación de un sector del mundo. Una teoría del derecho pretende ser una explicación de ese fenómeno de comunicación social que llamamos derecho. Pero, Raffaele De Giorgi, tal vez uno de los máximos responsables de la introducción de la teoría de la luhmanniana en la Argentina (junto a Javier Torres Nafarrate de México, a través de su traducción de las obras de Luhmann al español), pregunta: ¿Qué es una teoría de la sociedad? ¿Qué es el "Derecho de la sociedad"? y responde: es que podemos discutir esto?, manos que son manejadas por manos, nada diferente de una prótesis. Y agrega: estamos seguros de que los temas que usamos como objetos o los objetos que utilizamos como temas, son nuestra construcción, pensamos que nos volvemos sobre nosotros mismos, mientras continuamos girando alrededor del vacio.

Es una paradoja, u otra paradoja. ¿Es posible salir, deshacerse de estas paradojas? La lógica no nos ayuda. La lógica dice Luhmann, sólo puede ver el hecho de que algunas expresiones, cuya verdad no está disponible, entran en contradicción con otras expresiones, para las cuales vale decir la misma cosa. Usando operaciones lógicas, el problema no puede ser resuelto. Como terapia sistémica la única que podemos ver, con el auxilio

* Profesor titular Teoría general del derecho I y II, y director del Centro de Estudios Institucionales Patagónico (CEIP), Facultad de Derecho y Ciencias Sociales, Universidad Nacional del Comahue, Argentina, jorgedouglas952@gmail.com

PROBLEMA 
Esta revista forma parte del acervo de la Biblioteca Jurídica Virtual del Instituto de Investigaciones Jurídicas de la UNAM www.juridicas.unam.mx

http://biblio.juridicas.unam.mx

\author{
JORGE E. DOUGLAS PRICE
}

de la Gegenparadoxierungen ("contra-paradoja"), es un lugar menos doloroso en el que puede tolerarse el problema. Y recordó que, en cierto sentido, la colocación de la paradoja se realizará con más o menos éxito, esto es: de manera útil para desbloquear la situación.

$\mathrm{Y}$ es el uso creativo de paradojas, especialmente uno que se hizo por el Derecho, el que nos permite observar cómo su teoria (la de Luhmann) se ha comenzado a reciclar en las teorias existentes en Argentina. Temas como el problema de la paradojalidad del Derecho, de su ser sin fundamentos y sin comienzo, de la vacuidad de sus principios y la opacidad de sus operaciones y distinciones, se filtran teorías (como las de Carlos Cárcova o Ernesto Grün) y prácticas de la jurisprudencia y puede verse que ha comenzado a remover ese tipo de pensamiento que el profesor de Bielefeld había llamado "vétero europeo".

Palabras clave:

Niklas Luhmann, Raffaele De Giorgi, teoría sistémica, filosofia práctica en Argentina, Derecho y sociedad.

\begin{abstract}
:
A theory can be an explanation of a piece of the world. A theory of law is intended to be an explanation of that phenomenon of social communication which we call Law. But, Raffaele De Giorgi, perhaps the person responsible for the introduction of luhmannian theory in Argentina, (jointly with Torres Nafarrate in Mexico in translating Luhmann's work to Spanish), asked: What is a theory of society? What is the "Law of society"? and answered: Can we discuss this?, hands managed by hands, nothing different from a prostheses. According to De Giorgi we are sure that the themes that we use as objects or objects that we use as themes, are our construction, we think that we turn around ourselves, while we continue rotating around the vacuum. It is a paradox, or another paradox: ¿'Is it possible to get out or get rid of these paradoxes? Logic does not help us. Logic, according to Luhmann, can only see to the fact that some expressions, with unavailable truths, are in contradiction with other expressions, the problem cannot be resolved using logical operations. With the help of Gegenparadoxierungen (against-paradox), systemic therapy is the alternative where the problem can be tolerated. And, according to Luhmann, the placement of the paradox should be conducted in a more or less skilled, successful, or useful way to break the logjam.
\end{abstract}

A creative use of paradoxes, especially legal ones, allows us to observe how Luhmann's theory has impacted existing theories in Argentina.

PROBLEMA

Anuario de Filosofía y Teoría del Derecho,

Núm. 8, enero-diciembre de 2014, pp. 95-124 
Esta revista forma parte del acervo de la Biblioteca Jurídica Virtual del Instituto de Investigaciones Jurídicas de la UNAM www.juridicas.unam.mx

http://biblio.juridicas.unam.mx

TEORÍA DE LOS SISTEMAS DE NIKLAS LUHMANN

Themes such as the paradoxality of law, or its being without foundation (and without beginning, the vacuity of their principles and the opacity of its distinctions and operations), filtered theories (like Carlos Cárcova or Ernesto Grün) and jurisprudential practices have started shifting things to what Bielefeld Professor has called "old European".

Keyword:

Nuklas Luhmann, Raffaele De Giorgi, Systems Theory, Practical Philosophy in Argentina, Law and Society. 
Esta revista forma parte del acervo de la Biblioteca Jurídica Virtual del Instituto de Investigaciones Jurídicas de la UNAM www.juridicas.unam.mx

http://biblio.juridicas.unam.mx

JORGE E. DOUGLAS PRICE

En 1995 fue publicado en Argentina, por la Editorial Abeledo-Perrot, el libro "Una Visión Sistémica y Cibernética del Derecho", cuyo autor es el profesor Ernesto Grün, ${ }^{1}$ autor que continuó trabajando sobre el tema en publicaciones virtuales, especialmente en la Revista Telemática de Filosofia del Derecho, ${ }^{2}$ lo que le permitió publicar una segunda versión ampliada de su trabajo, en Editorial Dunken, en el año 2004 (Grün, Una visión sistémica y cibernética del derecho en el mundo globalizado del siglo XXI, 2004) y en el año 2006 publicó una tercera, en la Editorial Lexis Nexis (Grün, Una visión sistémica y cibernética del derecho en el mundo globalizado del siglo XXI, 2006).

El mismo año se llevó a cabo en la Universidad Nacional de Buenos Aires, organizado por el profesor Carlos María Cárcova, profesor de dicha Universidad, y referente principal (junto con Enrique Marí) de la vertiente de filosofia del derecho denominada "Teoría Crítica" en Argentina, el Congreso "El derecho en el fin de siglo", que contó con la presencia de numerosas figuras del campo internacional y nacional, tales como Alessandro Baratta, Joaquín Herrera Flores, José Eduardo Faría, Eros Grau, Ricardo Guibourg, el propio Carlos María Cárcova y el profesor italiano, Raffaele de Giorgi, de la entonces Universidad de Lecce (hoy del Salento), compañero intelectual de Niklas Luhmann en las dos últimas décadas de su vida y co-autor con el mismo Luhmann de la obra Teoría de la Sociedad, publicada en México.

También en 1995 el E. A. Russo publica su obra Teoría general del derecho. En la modernidad y en la posmoderni$\mathrm{dad},{ }^{3}$ y anuncia ya desde un comienzo que el punto a partir del cual su obra se esboza es el de la Teoría general de los sistemas de la sistemática social de Luhmann. En su opi-

1 Grün, Ernesto, Una visión sistémica y cibernética del derecho, Buenos Aires, Abeledo-Perrot, 1995.

2 Cfr. http://www.filosofiayderecho.com/rftd.

3 Cfr. Russo, Eduardo, Ángel, Teoria general del derecho. En la modernidad y en la posmodernidad, Buenos Aires, Abeledo-Perrot, 1995. 
Esta revista forma parte del acervo de la Biblioteca Jurídica Virtual del Instituto de Investigaciones Jurídicas de la UNAM www.juridicas.unam.mx

http://biblio.juridicas.unam.mx

TEORÍA DE LOS SISTEMAS DE NIKLAS LUHMANN

nión, dicha teoría, constituye uno de los avances epistemológicos más importante de los producidos en las últimas décadas del siglo XX, conjuntamente con los aportes de la semiología y de la lógica formal, conforman un tríptico indispensable para reflexionar sobre el pensamiento sistémico, dice expresamente:

La Teoría General de los Sistemas permitió pensar en metasistemas, así como la semiología descubrió la importancia del metalenguaje, y la pluralidad de lógicas formales, frente a la exclusividad de la lógica clásica, abrió la posibilidad de la metalógica. Sistemas que hablan de sistemas, lenguajes que hablan de lenguajes, lógicas que hablan de lógica, he ahí —afirma - la síntesis de la moderna epistemología. ${ }^{4}$

La obra de Russo si bien tiene un marcado tono ecléctico, y así lo anuncia, tiene el valor de formular una explicación e inserción de la teoría de los sistemas en la teoría general del derecho, que persigue la facilitación de su enseñanza en los cursos de grado de abogacía, precisamente en la Facultad de Derecho de la Universidad Nacional de Buenos Aires donde el autor enseñará hasta su fallecimiento ocurrido tempranamente en marzo de 2010. Señala que en el inicio de la teoría de los sistemas hay que colocar a la obra de Wolfgang Köhler5 (Teoría de la Forma, 1948) quien trasvasa el concepto de interacción utilizado en la física a la teoría psicológica, admitiendo que esa interacción es también un cierto recorte al mundo real, por cuanto la misma supone un cierto recorte del mundo real, desde que supone que para ser utilizada la interacción no es perturbada por factores externos, lo que permite vislumbrar el carácter constructivista, desde el punto de vista epistemológico de esta teoria, algo que va a estar presente de manera decisiva en la obra de Luhmann.

4 Idem.

5 Idem. 
Esta revista forma parte del acervo de la Biblioteca Jurídica Virtual del Instituto de Investigaciones Jurídicas de la UNAM www.juridicas.unam.mx

http://biblio.juridicas.unam.mx

JORGE E. DOUGLAS PRICE

Señala que casi simultáneamente con Köhler, Ludwig von Bertalanffy, comenzó elaborar la teoría de los sistemas abiertos, es decir la de aquellos sistemas que continuamente incorporan o eliminan datos o elementos, lo que modifica su estructura, no sólo su composición. Señala textualmente que:

Lo que en Köhler fue un trabajo de transposición de estructuras físicas a la psicología y a la biologia, en Bertalanffy se constituyó en un intento más ambicioso, que pudiese permitir, en definitiva tales transferencias de un sistema dado a cualquier otro... ${ }^{6}$

Russo también señala que un ejemplo de la utilización embrionaria de las teorias que alimentaron, a su vez a la teoria de Luhmann, se encuentra en la obra del Jerome Frank, quien al tratar sobre la teoría de la decisión judicial sostenía que:

la teoria tradicional simplifica al extremo, artificializa y distorsiona las verdaderas operaciones en que consiste la decisión de los tribunales...pues la reacción del tribunal no empieza frecuentemente con una neta diferenciación entre normas y hechos, sino con una reacción no analizada, indiferenciada, compuesta; una "corazonada", un 'gestalt' no analitico (un 'todo') ...cuando los jueces fallan sin dar opiniones o fundamento, se apoyan en sus 'gestalt' no analizados, y que aún cuando dan a publicidad sus opiniones o 'fundamentos' de hecho simplemente 'racionalizan' sus reacciones compuestas, sus intuitivas corazonadas o gestalts... tal reacción a menudo no es analítica, lógica, sino el resultado del juego de las influencias reciprocas de numerosas actitudes inconscientes... ${ }^{7}$

Advierte que el mismo Joseph Raz utiliza la noción de sistemas abiertos para referirse a la solución del problema

6 Ibidem, p. 215.

7 Idem.

PROBLEMA

Anuario de Filosofia y Teoría del Derecho, Núm. 8, enero-diciembre de 2014, pp. 95-124 
Esta revista forma parte del acervo de la Biblioteca Jurídica Virtual del Instituto de Investigaciones Jurídicas de la UNAM www.juridicas.unam.mx

http://biblio.juridicas.unam.mx

TEORÍA DE LOS SISTEMAS DE NIKLAS LUHMANN

presuntamente aporético de las "lagunas jurídicas", al afirmar que:

"Un sistema normativo es un sistema abierto en la medida que contiene normas cuyo propósito es dar fuerza vinculante dentro del sistema a normas que no pertenecen a él", que, agrega: "Cuantas más normas ajenas sean adoptadas por el sistema, más abierto es éste"(al sistema extrajurídico de entorno).

Es decir, señala Russo, que para Raz el entorno del sistema normativo está constituido por otros sistemas normativos extrajurídicos. ${ }^{8}$

El segundo elemento de la teoría proviene del campo de la lógica formal, sostiene, iniciada con la geometría euclidiana (siglo III a. C.), que constituye el primer sistema axiomático riguroso, sobre el que se desarrolló el modelo de sistema de enunciados por más de veinte siglos. El mismo Baruch Spinoza, señala Russo, trató de llevar esta idea al campo de la Ética, a través de su Ethica ordine geometrico demostrata, un intento al que, agrego, no es ajeno el mismo Hobbes en sus Elementos de Derecho Natural.

Sin embargo, prosigue, el profesor Russo, con el auge de las lógicas formales producido desde el comienzo del siglo, el tema de los sistemas axiomáticos volvió a ser la preocupación de los lógicos, se veía que la lógica aristotélica estaba, desde la perspectiva contemporánea, demasiado teñida de psicologismos y las deducciones no eran lo suficientemente rigurosas. Por ello, Carnap y Hilbert, procuraron precisar las nociones propias de los sistemas axiomáticos. Por ello, un sistema riguroso comienza construyendo un lenguaje propio, artificial, a la manera de los Principia Mathematica de Russell y Whitehead, a efectos de evitar las vaguedades y ambigüedades de los lenguajes naturales.

El tercer aporte provino del campo de la cibernética, desarrollado durante la segunda posguerra por Wiener, quien sostenía que "el propósito de la cibernética es desarrollar

$8 \quad$ Ibidem, p. 218. 
Esta revista forma parte del acervo de la Biblioteca Jurídica Virtual del Instituto de Investigaciones Jurídicas de la UNAM www.juridicas.unam.mx

http://biblio.juridicas.unam.mx

JORGE E. DOUGLAS PRICE

una lengua y unas técnicas que nos permitan afrontar no sólo los problemas más generales de comunicación y regulación, sino además establecer un repertorio adecuado de ideas y métodos para clarificar sus manifestaciones particulares de conceptos". En otros términos, señala Russo, el objeto de la cibernética es el estudio de los mensajes como medio de manejar aparatos o grupos humanos. Desde este punto de vista los conceptos de "lenguaje", "comunicación", "mensaje" y otros similares son "despsicologizados", restándoles todas las connotaciones propias de la comunicación humana. Ejemplo del desarrollo de la cibernética es la guía de misiles (con ella comenzó), o las células fotoeléctricas. El impulso inicial de este desarrollo hay que encontrarlo en la necesidad de desarrollar un lenguaje artificial de alta precisión para conectar hombres con máquinas.

Hace ver que frente a este panorama, que parece conducir a un cierto "melancólico" punto de vista filosófico, aparece la obra de Niklas Luhmann como un intento de construir una teoría sociológica unitaria, intento que Russo califica como de "racionalidad teleológica".

Tras señalar que observar cómo la teoría del derecho siguió los paradigmas científicos dominantes (tanto en el siglo XIX como en el XX, particularmente visible con el caso de Kelsen), señala Russo que la crítica a la teoría dogmática dominante (descendiente del modelo napoleónico, basado en la idea del modelo deductivo) hizo ver la crisis en tres frentes: a) las contradicciones internas (lagunas del derecho, abuso del derecho, vigencia de preceptos inconstitucionales, carácter no-lógico formal de las normas derogatorias, etcétera); b) la paradójica incapacidad para dar cuenta de ciertos fenómenos cotidianos (movimientos sociales, emergencias económicas, desobediencia civil, objeción de conciencia, función creadora de los jueces, etc.); y c) la evidencia de la falsedad de la supuesta neutralidad valorativa de ciertas instituciones o conceptos básicos (legitimidad, justificación de las formas de gobierno, justificación del mono- 
Esta revista forma parte del acervo de la Biblioteca Jurídica Virtual del Instituto de Investigaciones Jurídicas de la UNAM www.juridicas.unam.mx

http://biblio.juridicas.unam.mx

TEORÍA DE LOS SISTEMAS DE NIKLAS LUHMANN

polio del uso de la fuerza y del castigo, vigencia de los derechos humanos, etcétera). ${ }^{9}$

Según Russo la obra de Luhmann se nutre de un tríptico conceptual formado por la Teoría Crítica (Horkheimer, Adorno, Benjamín, el primer Fromm y Marcuse), la Teoría sociológica de Talcott Parsons (de quien fue discipulo), bien que disintiendo con éste en cuanto a que privilegia la función sobre la estructura y, finalmente, la teoría de los sistemas (von Bertalanffy, Ashby, Klir), aún si no le otorga a esta la unidad que puede presuponer su nombre. Para Luhmann, una teoría de los sistemas no puede ser sino "una teoría de los sistemas autorreferentes", lo que caracteriza al sistema no es su propio orden, sino su capacidad de reducir la complejidad del entorno, por ello el sistema aparece como un determinante de sentidos, como un mecanismo de selección que se vincula con la finalidad del sistema, y cita a Luhmann cuando dice:

Un dato real, que en el entorno posee una polisemia infinita $y$, por ello, inaprensible, resulta significativo por su incorporación dentro del sistema en cuestión, merced al rescate de algunos sentidos privilegiados en relación con el todo". Los sistemas sociales mediante su sentido constituyen simultáneamente sus limites y sus posibilidades de atribución de acciones. Esta atribución no necesita ser exclusiva. Un empleado que come su emparedado en el trabajo actúa en el sistema de la administración estatal y también en el sistema de familia. ${ }^{10}$

Simultáneamente con el de Russo aparece en la Argentina la obra del Profesor Ernesto Grün, quien desde su introducción plantea la nueva "Weltanschauung" que supone el enfoque sistémico, como sistema de conceptos, cuerpo teórico y una teoría de la praxis y metodologias de investigación, planificación y diseño de sistemas, destacando que la

9 Ibidem, pp. 267-268.

10 Luhmann, Niklas, Mustración sociológica y Otros Ensayos, Buenos Aires, SUR, 1973, p. 147. 
Esta revista forma parte del acervo de la Biblioteca Jurídica Virtual del Instituto de Investigaciones Jurídicas de la UNAM www.juridicas.unam.mx

http://biblio.juridicas.unam.mx

JORGE E. DOUGLAS PRICE

misma se inicia en la década de los treinta del siglo XX, con los estudios ya mencionados de Bertalanffy, a quien cita para señalar que:

Se trata de una construcción teórica que se ocupa de los principios y de las leyes que conciernen a toda clase de sistemas, en no importa qué rama científica, y que busca la formalización matemática de las relaciones y de las funciones isomorfas características del conjunto de los sistemas del mundo material e inmaterial. ${ }^{11}$

Tras destacar el cambio paradigmático, en el sentido kuhniano del término, que esta teoría supone, destaca que la misma importa un salto cualitativo que supera tanto al reduccionismo de la ciencia clásica (en tanto de pretensión de reducir todo a elementos últimos), como al método analítico (en cuanto postulaba la necesidad de "desarmar" en partes discretas a un todo que se suponía homogéneo) y al determinismo (que pretendia explicar todos los fenómenos como sucesiones de causa y efecto).

Señala Grün que finalizada la segunda guerra mundial y con los aportes realizados por los estudios científicos durante la misma se lleva a ver que cuando se "analiza" un sistema sus propiedades se pierden y comenzaron a utilizar la noción de función en lugar de la de estructura, esto es, dicho en sus propios términos:

Más que el por qué un sistema funciona de una cierta manera, para qué lo hace. Cabe advertir que ello no implica desechar el método analítico. Análisis y sintesis son complementarios y el pensamiento sistémico los incluye a ambos. ${ }^{12}$

Grün utiliza el concepto de sistema de Charles François quien lo caracteriza como:

11 Von Bertalanffy, Ludwig, Teoría general de los sistemas, México, Fondo de Cultura Económica, 1984.

12 Grün, Ernesto, Una Visión sistémica y cibernética del derecho en el mundo globalizado del siglo XXI, Buenos Aires, Dunken, 2004, pp. 23-24. 
Esta revista forma parte del acervo de la Biblioteca Jurídica Virtual del Instituto de Investigaciones Jurídicas de la UNAM www.juridicas.unam.mx

http://biblio.juridicas.unam.mx

TEORÍA DE LOS SISTEMAS DE NIKLAS LUHMANN

Una entidad autónoma dotada de una cierta permanencia y constituida por elementos interrelacionados que forman subsistemas estructurales y funcionales, que se transforma dentro de ciertos límites de estabilidad, gracias a regulaciones internas que le permiten adaptarse a las variaciones de su entorno específico. ${ }^{13}$

La piedra angular, afirma, de la existencia de todo sistema es el hecho de que está aislado, pero su aislamiento no es absoluto, aunque sí lo suficiente como para poder ser distinguido de su entorno, clara y permanentemente. El sistema, en el caso de los sistemas biológicos, está separado del entorno por un límite o membrana que separa "al endomundo del exomundo". Esta frontera, continúa, no es siempre fácil de identificar y, en muchos casos, depende de decisiones, por lo menos lingüísticas y casi siempre pragmáticas, de donde deduce esta afirmación:

la constitución del sistema depende del observador y de sus decisiones metodológicas. ${ }^{14}$

Para el mantenimiento de su identidad (o unidad en un sentido más clásico), el sistema o el metasistema viviente necesitan de un dispositivo regulador - por lo general complejo- centrado en su mecanismo de observación, de control y de reproducción de sus propias estructuras. Continúa refiriendo Grün que todo sistema complejo (y los seres humanos y los sistemas sociales lo son) es parte cambiante de una totalidad más vasta que lleva hasta el sistema dinámico más complejo de todos que es el universo mismo.

Señala que un ingrediente clave son las interacciones no-lineales entre sus componentes, las que, bajo circunstancias especiales, pueden originar conductas emergentes complejas con una estructura muy rica. Esto es lo que se indica cuando se indica que el todo es más que la suma de las partes. El sistema tiene una forma Gestalt y esta puede

13 Ibidem, p. 29.

14 Ibidem, p. 31. 
Esta revista forma parte del acervo de la Biblioteca Jurídica Virtual del Instituto de Investigaciones Jurídicas de la UNAM www.juridicas.unam.mx

http://biblio.juridicas.unam.mx

JORGE E. DOUGLAS PRICE

ser caracterizada como la configuración de un grupo de elementos percibido como una totalidad organizada, las partes no existen previamente al todo sino que derivan su carácter de la estructura del todo. ${ }^{15}$ Esto lo lleva a hacer notar la importancia de la noción de modelo, en tanto que el modelo permite reflejar la naturaleza funcional-dinámica de los sistemas reales. ${ }^{16}$ Él previamente ha señalado que la realidad presenta dos aspectos básicos inseparables: lo estructuralestático y lo funcional-dinámico, análisis que recuerda, me permito agregar, al modelo de análisis del sistema jurídico realizado por Kelsen.

Tras describir, lo que entiende como descripciones fallidas o parciales del sistema del derecho, formuladas por Kelsen, Ross y Hart (incluyendo la crítica de Cárcova al primero de ellos por su reduccionismo normativista) y apuntar las críticas del tridimensionalismo (que tuviera cultores en la Argentina como Carlos Cossio, Julio Cueto Rúa y Werner Goldschmidt entre otros, y en Brasil a la figura de Miguel Reale), apunta que la crítica de Rudolph von Ihering a fines del siglo XIX reclamaba ya el paso del concepto de sistema de derecho al de sistema parcial de la sociedad como el mismo Luhmann afirmara. ${ }^{17}$

Tras ello, Grün repasa los enfoques que denomina sistémico-cibernéticos del derecho, para lo que analiza la concepción de Kelsen, anotando el giro hacia cierto monismo internacional, como producto de la globalización (aspecto que Kelsen ya había anticipado como posible), señala que una importante corriente de autores basados en las teorias de Niklas Luhmann (que a su vez se sustenta en la teoría de la autopoiésis de los biólogos chilenos Maturana y Varela), consideran al sistema jurídico como autopoiético y en consecuencia como prácticamente cerrado. Si bien refiere Grün la principalidad de la noción de autopoiésis en la teoría de Luhmann, advierte que es un concepto de difícil de-

15 Idem.

16 Ibidem, pp. 32-33.

17 Ibidem, p. 45.

PROBLEMA

Anuario de Filosofia y Teoria del Derecho, Núm. 8, enero-diciembre de 2014, pp. 95-124 
Esta revista forma parte del acervo de la Biblioteca Jurídica Virtual del Instituto de Investigaciones Jurídicas de la UNAM www.juridicas.unam.mx

http://biblio.juridicas.unam.mx

TEORÍA DE LOS SISTEMAS DE NIKLAS LUHMANN

sarrollo, que el sociólogo alemán utiliza para caracterizar a los sistemas sociales, como el derecho. Revela sí que lo sistemas autopoiéticos no tienen "inputs" ni "outputs", que sólo reciben "shocks" exógenos, perturbaciones, pero — sostiene- estos no actúan como información que contribuya a programar al sistema, sino que sólo provoca reacciones compensatorias internas, para mantener invariable el equilibrio homeostático del sistema. Así el autor señala a partir de una serie de "leading cases" de la Corte Suprema de Justicia de la Argentina como se produce el proceso de feedback o retroalimentación en un sistema jurídico. ${ }^{18}$

Carlos María Cárcova uno de los más brillantes filósofos del derecho de la Argentina, titular de la cátedra de Teoría General del Derecho de la Universidad de Buenos Aires y actual Director del Instituto de Investigaciones Jurídicas "Ambrosio Gioja", de la misma Universidad introduce en su libro "La opacidad del Derecho". Tras señalar que la teoría de Luhmann presenta un entramado conceptual altamente abstracto, frecuentemente mediado por consideraciones indiferentes a cualquier clase de compromiso ético, que le valieran por parte de alguno de sus adversarios el mote de "Maquiavelo de la sociedad del capitalismo avanzado", advierte que sus aportaciones son de una importancia y originalidad poco frecuentes. ${ }^{19}$

Afirma Cárcova que para este autor, el derecho es el resultado de un azaroso proceso evolutivo; proceso que es consecuencia, a su vez, de la complejidad y contingencia del mundo. La complejidad está dada, según Luhmann, por el "exceso de posibilidades del mundo" y la contingencia por la imprevisibilidad y variabilidad de tales posibilidades. Ello, continúa explicando Cárcova: "implica una sobre-exigencia para la interacción social, que tiende a resolverse mediante la reducción de la complejidad operada a través del surgimiento y la operatividad de distintos subsistemas

18 Ibidem, pp. 51-55.

19 Cárcova, Carlos María, La opacidad del derecho, Madrid, Trotta, 1998, p. 33. 
Esta revista forma parte del acervo de la Biblioteca Jurídica Virtual del Instituto de Investigaciones Jurídicas de la UNAM www.juridicas.unam.mx

http://biblio.juridicas.unam.mx

JORGE E. DOUGLAS PRICE

sociales. Señala que la aparición de los subsistemas sociales, entre ellos el derecho, da cuenta del tratamiento de la complejidad del ambiente, así — por ejemplo, afirma- que en el mundo romano bastaba con la diferenciación entre iusquiritiumy iusgentium, para atender la variabilidad de las relaciones sociales, pero con la evolución de la sociedad y su progresiva complejización, el derecho se va fragmentando y especializando, es decir: se va diferenciando, y esto permite dar cuenta de relaciones sociales más complejas y variables. ${ }^{20}$

Para Luhmann, dice Cárcova, el derecho tiene como función la generalización congruente de expectativas de conducta, a través de las cuales reduce la complejidad. Se trata de un efecto producido por la representación del sistema de normas. Luhman, dice Cárcova:

Sintetiza de este modo el fenómeno que Weber caracterizara como el "desencanto del orden", en el sentido de que su fundamento pasa del lugar del mito al de la religión, de ésta al de la razón universal, para concluir, al fin, en un artificio lógico o ficcional, en un reconocimiento o consenso que también puede ser real o ficcional. En todo caso, el fenómeno de la positivación del derecho implica, en las sociedades modernas, efectos conspicuos en tres dimensiones diferenciadas: en la dimensión temporal, mediante la utilización de sanciones a través de las cuales se absorberian las frustraciones del orden o, en una terminología más próxima, la eventual inobservancia de la norma por parte del súbdito; en la dimensión social, mediante la producción de consenso a través de la ampliación de los procedimientos socialmente vinculantes en la toma de decisiones acerca del derecho; en la dimensión material, mediante la diferenciación de roles entre quienes aplican el derecho y quienes establecen los programas a aplicar. En otras palabras entre jueces y legisladores. ${ }^{21}$

20 Idem.

21 Ibidem, p. 35.

PROBLEMA

Anuario de Filosofia y Teoría del Derecho, Núm. 8, enero-diciembre de 2014, pp. 95-124 
Esta revista forma parte del acervo de la Biblioteca Jurídica Virtual del Instituto de Investigaciones Jurídicas de la UNAM www.juridicas.unam.mx

http://biblio.juridicas.unam.mx

TEORÍA DE LOS SISTEMAS DE NIKLAS LUHMANN

El siguiente paso de la introducción de la Teoría en la Argentina fue dado por el mismo Raffaele de Giorgi, quien comenzó a partir del año 2000 a dictar diversos seminarios en la Universidad Nacional del Comahue (norte de la Patagonia), coordinados por el autor de este artículo.

El doctor Raffaele de Giorgi se formó, en su etapa de posgraduación, en Alemania, donde estudió filosofia jurídica y social, fue colaborador de Niklas Luhmann a partir de 1979, con quien publicó el libro, y referido, Teoría de la sociedad y con quien fundará, en 1990, en la Universidad de Lecce (hoy del Salento) el Centro de Estudios sobre el Riesgo, que dirige hasta el presente. La finalidad del Centro ha sido definida como del estudio del riesgo en la sociedad contemporánea. Con ello se propone observar y describir, mediante análisis teóricos y empíricos, los modos según los cuales al actuar fáctico puede ser condicionado por decisiones (como las del derecho) que producen vínculos con el futuro, porque se asumen en condiciones de incerteza; de aislar temas específicos del actuar social a través de la referencia a ámbitos de problemas delimitados; de tematizar, a través de investigaciones específicas, la globalidad del tema del "riesgo"; de observar y describir las transformaciones estructurales que se producen en la sociedad contemporánea, en la cual el riesgo ha devenido tema de la comunicación social como derivación del desarrollo y la difusión de la tecnologia; de proveer, en suma, resultados capaces de esclarecer la particularidad de la perspectiva "riesgo-peligro", a través de la reconstrucción semántica de los conceptos no sólo de riesgo y de peligro, sino también de incerteza, inseguridad, daño, incidente, que a menudo son considerados solo como simples variantes semánticas de la palabra "riesgo".

Las investigaciones conducidas en el Centro de Estudios sobre el Riesgo, a menudo interdisciplinarias, siguen derroteros comunes sobre los temas de la percepción, clasificación, valoración y tratamiento de los riesgos, pero si diferencian por sus específicos ámbitos disciplinarios, como la 
Esta revista forma parte del acervo de la Biblioteca Jurídica Virtual del Instituto de Investigaciones Jurídicas de la UNAM www.juridicas.unam.mx

http://biblio.juridicas.unam.mx

JORGE E. DOUGLAS PRICE

economía, la política, la ciencia, la tecnología, el derecho, con el fin de mostrar, y describir las características de los mecanismo que cada uno de los sistemas sociales activa al confrontar el problema del tratamiento de la incerteza. El Centro es también sede del Doctorado de Investigación Jurídica sobre "Evolución de los sistemas jurídicos y nuevos derechos".

En 1997, de Giorgi ya había coordinado un seminario en la Universidad Nacional Autónoma de México (UNAM), denominado "La Construcción Social de la Legalidad en México", auspiciado por la Dirección General de Asuntos del Personal Académico de la UNAM y el Consejo Nacional de Ciencia y Técnica, en compañia de los profesores Fernando Castañeda Sabido y Angélica Cuellar Vázquez, encuentro en el que se intercambiaron experiencias en torno al tema de la autoridad pública, aunque a lo largo de su desarrollo, se introdujeron temas que enriquecieron el debate, tanto en el plano teórico como en el de la praxis inmediata.

En la investigación específica, que cito a modo de ejemplo, conducida por el Profesor de Giorgi, se investigó -a partir de datos de campo- la estructura de los comportamiento sociales y que de hecho llevan a la deslegitimación de la misma normatividad jurídica, se trata, decía:

...de estructuras de la autoridad que tienen relevancia pública, pero que, precisamente por esto la debilitan, la desnaturalizan...Estas estructuras son visibles, son organizadas, utilizan el reconocimiento jurídico, pero se imponen a sí mismas prácticas alternativas al derecho, maltratan el derecho y evidencian otros recorridos del poder que son alternativos a los recorridos del poder que se realizan a través del derecho porque son controlados por el derecho. ${ }^{22}$

El autor de este artículo participó de dicho evento y en su trabajo puntualizaba que en los países periféricos se construyen distinciones (inclusiones-exclusiones) a través de las

22 Giorgi, Raffaele de, "Introducción", Redes de inclusión - La construcción social de la autoridad, México, Porrúa, 1998. 
Esta revista forma parte del acervo de la Biblioteca Jurídica Virtual del Instituto de Investigaciones Jurídicas de la UNAM www.juridicas.unam.mx

http://biblio.juridicas.unam.mx

TEORÍA DE LOS SISTEMAS DE NIKLAS LUHMANN

que denominará, siguiendo a de Giorgi, como "redes informales de la dependencia", en las que coexisten una gran formalidad en el decidir con una alta informalidad en la constitución social de la autoridad, que convierte a las distinciones jurídicas en indistintas, vagas e inciertas:

El supuesto es paradojal: bajo las formas de la modernidad, en específico las formas del derecho, o sus procedimientos racionales, existen en nuestras sociedades, que los autores llaman "periféricas", otros códigos que inhiben la racionalidad de los procedimientos del derecho y producen autoridad, lo que lleva a observar que "a través de redes informales de dependencia" se hace posible que la más alta formalización del decidir pueda coexistir con la más alta informalidad de la constitución de la autoridad. ${ }^{23}$

A partir de entonces realizó con el Profesor de Giorgi una serie de seminarios en la Universidad Nacional del Comahue (Norte de la Patagonia), en la Universidad de la Punta (San Luis) y en la Universidad Nacional de Buenos Aires (UBA), en los años 2000 (sobre "Sociología del Riesgo"), 2008 ("La decisión judicial en el sistema jurídico contemporáneo"), 2010 (Sobre "Federalismo, Derechos Humanos y Diversidad"), con intervenciones de otros destacados profesores como Santos Colabella, Juan Manuel Salgado, Cristina García Vázquez, José M. Belisle de Argentina; Guilherme Figueiredo Leite Gonçalves, de Brasil y Ada Przreni, de Albania, entre otros).

De Giorgi señalaba, en el primero de los mencionados seminarios, recordando la obra homónima de Luhmann, que el umbral del riesgo puede fijarse de una manera muy diversa, según sea el caso de que uno participe del riesgo como portador de decisiones o como afectado por las decisiones riesgosas, por lo que es difícil pensar que los cálcu-

23 Douglas Price, Jorge Eduardo, "Democracia, poder local y globalización. ¿Es posible constituir poderes locales autónomos?”, en varios autores, Redes de inclusión. La construcción social de la autoridad, México, Porrúa, 1998. 
Esta revista forma parte del acervo de la Biblioteca Jurídica Virtual del Instituto de Investigaciones Jurídicas de la UNAM www.juridicas.unam.mx

http://biblio.juridicas.unam.mx

JORGE E. DOUGLAS PRICE

los de riesgo tengan posibilidades de despertar consenso, inclusive en el caso de situaciones específicas. Que tal como lo han señalado los antropólogos culturales y sociales, la evaluación del riesgo y la disposición a aceptarlo no es sólo un problema psíquico sino también social. Uno se comporta tal como lo esperan los grupos de referencia relevantes, o tal como uno ha sido socializado (sea de acuerdo con la opinión comúnmente aceptada o en contra de ella). ${ }^{24}$ Pero ello nos lleva a preguntarnos cómo debe estar conformada una comunicación debe estar conformada una comunicación que pretende elevar la conciencia del riesgo (y tenemos presente que para Luhmann la sociedad son las comunicaciones que los aparatos psíquicos producen).

Desde esta perspectiva es que se analizó en 2008 el rol de los tribunales en el sistema del derecho, en el que el Profesor de la Fundación Getulio Vargas, Figueiredo Leite Gonçalves, señalaba como la Constitución es el punto de diferenciación entre Derecho y Politica, el punto en el cual el sistema del derecho puede autonomizarse, a partir de diferencias tales como la de "jurisprudencia" y "legislación" y cómo ésta diferencia se mantiene siempre en condiciones de alta inestabilidad que amenazan con hacerla desaparecer.

Y, con una imagen de Hofstadter, de Giorgi dice que el sistema debe ser visto:

como una jerarquía intrincada en la cual se han condensado los rastros, los residuos, los fósiles que la evolución del derecho a sedimentado a lo largo de su recorrido. Si el derecho moderno, que es derecho plenamente positivo, ha abandonado en la actualidad el uso de viejas distinciones, todavia, en la autorrepresentación universalizante de la Jurisprudencia, muchas de aquellas distinciones continúan celebrando su resistencia. Piénsese por ejemplo en distinciones como ley y derecho, naturaleza y razón, naturaleza y cultura, hecho y valor, ser y deber ser, realidad y construcción.

24 Luhmann, Niklas, Sociología del riesgo, Guadalajara, Universidad de Guadalajara-Universidad Iberoamericana, 1992. 
La Jurisprudencia es la jerarquía intrincada que las conserva y que periódicamente las reactiva, aunque sí en modo claramente contrafáctico. En esta intrincada jerarquía se forman "extraños bucles": en el lenguaje de Hofstadter extraño bucle indica "alguna cosa que está dentro del sistema, sale del sistema e incide en el sistema, como si estuviera fuera del sistema.

La evolución del derecho produce mutaciones semánticas en virtud de las cuales la paradoja de la autoproducción del derecho se confunde con los extraños anillos de la Jurisprudencia, se dispersa en sus complejidades. Las mutaciones recientes de la Jurisprudencia se llaman Jurisprudencia de conceptos, Jurisprudencia de intereses, Jurisprudencia realista, dogmática, progresista, conservadora, Jurisprudencia liberal, crítica, alternativa. Como efecto de estas mutaciones se forman retículas conceptuales que sirven como fósiles guías en la orientación de la actividad jurisprudencial; la cual consiste siempre en la producción de conceptos sobre la base de conceptos, en la construcción de distinciones en base a distinciones, en la sedimentación de sentido en base a sentido. En las operaciones, sin embargo, la estratificación semántica que es puesta en acción debe presentarse de manera que la recursiva actualización de las distinciones interrumpa la construcción lógica, o también la idea de intereses, o la valoración realista de las condiciones sociales en las cuales se producen los acontecimientos, o el fin, esto es, las consecuencias calculables de la construcción jurisprudencial. Puntos de vista internos del sistema resultan aislados mediante el uso de distinciones que luego son reintroducidas en el sistema como orientación y guía de la construcción de otras distinciones, con las cuales el sistema se asegura su capacidad de continuar operando. 25

Finalmente, pero no por último, en el año 2012, el autor de este artículo publicó, dentro de la línea de investigación iniciada en el referido doctorado de la Universidad de Lecce,

25 Giorgi, Raffaele de, Jurisprudencia, Campeche, Corte Suprema de Justicia de México, 2008. 
entre los años 2001 y 2006, su libro "La Decisión Judi cial", ${ }^{26}$ en el que resume su propósito de este modo:

...es común que la Teoría General del Derecho analice el problema de la decisión judicial desde la decisión misma, como silogismo, como pura decisión o como cadena de argumentos. Pero, además, la decisión judicial puede ser vista como una función del sistema, esto es: como una operación de la cadena de operaciones que ha permitido al sistema diferenciarse.

Esto significa considerar - afirma- al derecho tanto como un sistema de enunciados cuanto que como un sistema social, es decir: como una cadena de operaciones comunicacionales, y en tanto tales como acciones sociales típicas, compartiendo pues con Luhmann que el del derecho, como todos los subsistemas de la sociedad, está constituido por ellas.

En este sentido, adelanto, comparto también con Luhmann que el sistema de derecho funciona como tal, es decir, como un sistema autónomo, pero - vaya aquí una primera y tal vez decisiva advertencia - su programa decisional solo asegura que tomará decisiones, no cuáles decisiones.

Si se adoptan siguiendo un procedimiento (o no), y si ese procedimiento (cuando se sigue) supone implícitos (o no) una cierta lógica y/o unos ciertos contenidos o soluciones restringidas (al menos en el "elenco") lógica o axiológicamente, es tema que analizaré más adelante.

Es decir, desde una perspectiva, la del derecho como sistema de comunicaciones, el programa del derecho asegura que los jueces decidan, no cómo vayan a decidir ni qué van a decidir.

Por otra parte: en el interior de las mismas decisiones, donde problemas como racionalidad e irracionalidad aparecen, sostengo que apenas puede pretenderse asegurar que esas decisiones guarden coherencia con algún principio elegido de entre un catálogo de principios o lugares comunes ("topoi" en la terminología aristotélica) creados por la doctrina (académica o judicial), no todos ellos jurídicos "stricto

26 Cfr. Douglas Price, Jorge Eduardo, La decisión judicial, Santa Fe, Argentina, Rubinzal Culzoni, 2012. 
sensu", o, recordando las lecciones de Raffaele de Giorgi, jurídicos porque no son jurídicos, transformados en jurídicos por la misma operación que transforma en jurídico todo lo que el sistema del derecho toca (ya Kelsen habló del derecho como Midas), lo que por ende deja el catálogo "abierto".

Si bien la lógica clásica no parece ser la herramienta de las decisiones, más que en un limitado sentido, con Tecla Mazzarese repasaré la posibilidad de que la racionalidad del método decisorio pueda estar controlada por algún otro tipo de lógica, tal como - por ejemplo- la llamada lógica fuzzy, difusa o borrosa. Este trabajo admite que una lógica tal pueda llegar a ser usada, pero que tampoco es un recurso actual del sistema.

El sistema, sostengo, no asegura ni la corrección de los puntos de partida fácticos (determinación de los hechos) -que están apenas controlados por ciertos criterios provenientes de las ciencias experimentales ${ }^{27}$ y más débilmente por la denominada "ciencia procesal" - ni la validez de los topoi jurídicos, a los que presupone válidos dogmáticamente, ni mucho menos los criterios de selección de las referencias de valoración que también quedan reservados al decisor por esa misma "ciencia".

Es que si bien el sistema presupone "arrancar" ex nihilo en el instante constitucional, ${ }^{28}$ el instante de la diferenciación entre política y derecho, dejando fijados los parámetros básicos de Justicia; no está — sin embargo- en condiciones de regular o controlar cuál será el principio, regla, o topoi que seleccionará el sistema decisorio judicial antes de resolver cada caso. Sabe apenas que seleccionará uno de entre varios, en un catálogo que es móvil y que se está expandiendo entrópicamente. ${ }^{29}$

Por lo tanto, vale advertirlo, ese punto es un punto móvil, contingente, el contenido constitucional puede ser variado en cada momento por el mismo procedimiento con que fue

27 De extrema labilidad en este campo.

28 Se introduce aquí toda la problemática tratada por Kelsen en su teoría de la Grundnorm.

29 Tengo en mente aquí los problemas derivados de la expansión de los derechos a nivel de constituciones y tratados, que ha llevado al debate sobre el minimalismo constitucional. 
instaurado, lo cual es obvio, pues el sistema de control constitucional que contiene como presupuesto de restricción, paradójicamente, permite hacerlo fluctuar a partir del método de interpretación apoyado en el de argumentación. Pero, a su vez, esto debe ser visto como el punto clave de su éxito: como factor de estabilización del sistema; una mayor rigidez lo inmovilizaría al punto de hacerlo implosionar.

Sostengo, sin embargo, que dos principios deberá sostener el sistema jurídico en sus operaciones, desde el punto de vista interno: el de congruencia (al interior de la decisión) y desde el punto de vista externo: el de la analogía (aunque sólo en relación a otras decisiones, que no quiere decir -ni podría decir - a todas las otras), para lo cual nos resultará sumamente útil ver el funcionamiento concreto de la regla del stare decisis en el commonlaw.

Convengo en que afirmar esto, aún con los matices apuntados, supone una restricción al discrecionalismo absoluto y que esta es precisamente la adquisición evolutiva del derecho: "su separación de la política a partir de la instauración del mismo procedimiento que lo caracteriza como sistema, ${ }^{30}$ la resolución del conflicto a partir de la analogía, marca una fuerte restricción a la discreción, que a su vez marca un punto de diferenciación con los restantes sistemas de control social, esto no se invalida porque los desarrollos en tal sentido del derecho griego y del romano se hayan prolongado en el derecho canónico, desde que éste no es sino la expresión de una moral secularizada y en este sentido transformada en derecho. Qué cual sea la posible evolución del sistema del derecho moderno en el siglo XXI, es cuestión indudablemente conectada a la respuestas que se den a estos problemas.

Es así, continúa, que podemos ver entonces al sistema del derecho como un sistema autopoiético, como un siste-

30 En este sentido podría verse ya una primera diferenciación con la prohibición de los juicios de Dios u Ordalías, expresada por la iglesia católica en la Baja Edad Media (circa 1123, año del Concilio de Works). Las costumbres medievales, establecidas bajo el influjo directo de los pueblos "bárbaros", empezaban a modificarse. Pascual II, intervino para que se suprimiese la práctica de la ordalía o juicio de Dios. Contra el duelo judicial se pronunció Nicolás I y fue combatido por los Papas de la época.

PROBLEMA

Anuario de Filosofia y Teoría del Derecho, Núm. 8, enero-diciembre de 2014, pp. 95-124 
Esta revista forma parte del acervo de la Biblioteca Jurídica Virtual del Instituto de Investigaciones Jurídicas de la UNAM www.juridicas.unam.mx

http://biblio.juridicas.unam.mx

TEORÍA DE LOS SISTEMAS DE NIKLAS LUHMANN

ma que se reproduce a sí mismo (esto ya había sido advertido por Kelsen al señalarlo como un sistema que regula su propia creación):

El derecho constituye un sistema autopoiético de segundo grado, en cuanto sistema autopoiético de primer grado, gracias a la constitución auto-referencial de sus propios componentes sistémicos y la articulación de éstos en un hiperciclo. ${ }^{31}$

Esta definición no está ciega a las críticas y dudas que albergan detractores y defensores de la tesis, preguntas del tipo: cómo, qué, o quién se produce a sí mismo, o cuál es el papel de los seres humanos, de los individuos en este proceso, se reiteran. Qué elementos del sistema, pregunta Teubner, juristas, organizaciones jurídicas, argumentación jurídica (agregaríamos, procedimientos, interpretación, etc.) se podrían considerar como reproduciéndose a sí mismas? ${ }^{32}$

Los seres humanos desempeñan un doble papel, responde, en este proceso, pues funcionan simultáneamente como "constructos semánticos" del sistema jurídico y como "sistemas psiquicos autopoiéticos independientes", pertenecientes al "medio ambiente" que rodea al propio sistema jurídico.

Esta visión es recusada por algunos como un nuevo tipo de formalismo, o una nueva ideología generada por los juristas, sin embargo, dice Teubner, esto es fácil de responder: autorreferencia y autopoiesis dan origen a nuevo y más elaborado tipo de autonomía del sistema jurídico, en virtud de la constitución de relaciones circulares, lo que no excluye sino que presume las relaciones de interdependencia entre sistema jurídico y sistema social. 33

Importante para observar el punto es advertir la réplica de Teubner a Luhmann en el sentido de advertir que puede haber autonomía o autopoiesis "parciales", mientras que

31 Teubner, Gunther, O Direito como sistema autopoiético, Lisboa, Fundação Calouste Gulbenkian, 1989.

32 Ibidem, p. 55.

33 Ibidem, p. 56. 
Esta revista forma parte del acervo de la Biblioteca Jurídica Virtual del Instituto de Investigaciones Jurídicas de la UNAM www.juridicas.unam.mx

http://biblio.juridicas.unam.mx

JORGE E. DOUGLAS PRICE

para el autor de Teoría de la Sociedad (como para Maturana) el concepto de autopoiesis era de una rigidez inflexible (o el derecho se reproduce a sí mismo o no se reproduce), para Teubner se podia introducir la idea formulada por Eigen y Schuster de "hiperciclo" (como principio de la auto-organización natural), diciendo que "un sistema juridico se torna autónomo en la medida en que consiga constituir sus elementos - acciones, normas, procesos, identidadesen ciclos autorreferenciales, solo alcanzando el término eficiente de su autonomía autopoiética cuando los componentes del sistema, así cíclicamente constituidos, se articulen entre sí mismos, a su vez, formando un hiperciclo"34

Está claro que las metáforas en búsqueda de la "explicación perdida" se solapan: "extraños bucles" (Hofstader/de Giorgi), "hiperciclo" (Eigen-Schuster/Teubner) o como propondré ahora, "nudo borromeo" (Lacan), lo cierto es que lo que se intenta explicar es la noción de un paradigma distinto sobre la constitución de lo "nuevo", es decir sobre la emergencia de la diversidad. Paradójicamente, tal vez, deberíamos quedarnos mudos, como sugería Kuhn, frente a la ausencia de un paradigma explicativo que reemplace a aquél que ha quedado en crisis. Sostengo, sin embargo, que estas metáforas explican mejor el surgimiento del sistema que la teológica (iusnaturalista) o la voluntarista (positivista), porque ambas padecen de insuficiencias explicativas que no podemos discutir aqui, pero que podrian resumirse en que la primera remite a un origen incomprobable per se y no explica la "diferencia" entre régimen y realidad, mientras que la segunda, si bien explica el origen en un acto de voluntad, también se ve precisada de hipostasiarla para explicar cómo funciona el cambio, y aún para explicar por qué la regla fundante se parece en mucho a la del Rey del famoso relato de Saint-Exúpery. ${ }^{35}$

34 Ibidem, p. 58.

35 Ante las respuestas espontáneas del pequeño Príncipe, quien se niega a bostezar, por timidez, el Rey sentencia: "Te ordeno tan pronto que bosteces y que no bosteces...”, Saint-Exúpery, Antoine, Le Petit Prince. 
Esta revista forma parte del acervo de la Biblioteca Jurídica Virtual del Instituto de Investigaciones Jurídicas de la UNAM www.juridicas.unam.mx

http://biblio.juridicas.unam.mx

TEORÍA DE LOS SISTEMAS DE NIKLAS LUHMANN

En el "nudo borromeo"36 hay partes en que un cordel pasa por encima de otro (supeditándolo localmente) y luego, en otra, la relación se invierte y el que pasaba por arriba (overcrossing) pasa por debajo (undercrossing); de tal modo se alternan los distintos registros sin conformar una estructura jerárquica. Se trata de "vínculos" que se "anudan" de tal suerte que cortando uno, los otros se desatan, se "des-anudan", dejando nuda la patética "realidad" de lo humano: estamos "sujetos" por nudos inseguros, tales que si uno de los lazos se cortaran, los demás se "des-anudarian".

La teoría, se teje y desteje como la tela de Penélope. Como decían el mismo Luhmann y de Giorgi, en Teoría de la Sociedad, el objeto de su investigación es el sistema social de la sociedad moderna, lo que presenta numerosas dificultades, entre ellas que el término "sociedad" no se asocia con una representación unívoca, ni tampoco el término "social" presenta referencias objetivas. Además, señalaban, el intento de describir la sociedad puede efectuarse sólo en la sociedad, es decir, hacer uso de la comunicación (de la que está constituida la sociedad), con lo que activa relaciones sociales y se expone a la observación. Entonces, de cualquier manera que se pretenda definir al objeto, la definición misma es ya una operación del objeto. La descripción realiza lo descripto: en el momento en que tiene lugar tiene que describirse a sí misma, es decir: manos que dibujan manos, como las sentencias judiciales. ${ }^{37}$

En suma, como refiere, la Profesora de la Universidad de Buenos Aires, Nora Wolfzun, en un reciente artículo publicado en la Revista de Filosofia del Derecho del Ministerio de Justicia y Derechos Humanos de la Nación:

36 Me inspiro en la célebre idea de Jacques Lacan, solo en la modesta aproximación que una metáfora produce sobre un lector (con sus infinitas derivas), no pretendiendo dejar por ahora más que un boceto sobre el que pretenderé volver en el futuro, cuando el conocimiento de su obra, así me lo permita.

37 Cfr. Luhmann, Niklas y Giorgi, Raffaele de, Teoria de la Sociedad, Guadalajara, Universidad de Guadalajara-Universidad Iberoamericana, 1993. 
Para una perspectiva critica del derecho, la teoría de sistemas autopoiéticos de Niklas Luhmann aporta una saludable tensión que coadyuva a subvertir algunos esquemas conceptuales y analíticos claves, naturalizados y enquistados en el sistema jurídico por enfoques más reduccionistas o más estáticos. Nociones como autopoiesis, circularidad, paradoja, recursividad, contingencia, complejidad y otras, conmueven los andamiajes de un pensamiento dogmático y formal para encarnarse estructuralmente, al decir de su discípulo Gunther Teubner (1995), en la realidad social del derecho. ${ }^{38}$

Sostiene Wolfzun que la teoria autorreferencial de Luhmann ofrece herramientas para desmitificar el derecho como 'el' discurso de 'la' verdad, crítica que se vuelve necesaria dado el carácter organizador del discurso social sobre el que se funda la distribución de bienes y cargas (lo que podríamos llamar una teoría de la justicia, sin resonancias esencialistas) en la sociedad contemporánea.

Agrega que, desde una perspectiva crítica y constructivista:

los hechos y las normas no son más (ni menos) que artefactos construidos hacia el interior del sistema jurídico, a partir de una lógica propia y de espaldas a cualquier aproximación ontológica, creando al mismo tiempo campos de sensibilidad y de indiferencia, y nada existe por fuera de su compleja red de operaciones selectivas. El derecho, tal como cada sistema lo hace, construye su propia realidad a la medida de sus necesidades. Es soberano en la atribución de sentido, en la construcción de identidades, ficciones, clasificaciones, relaciones, diferencias. Y si a esto sumamos la idea del derecho como discurso del poder (y al Estado como detentador legitimo del monopolio de la fuerza), comprendemos la enorme tentación de lo jurídico por pretender un universalismo incuestionado acerca de su mirada sobre el mundo social. Como afirma Nelson Goodman (1990: 22), la realidad como artificio invita a pensar en muchos mundos posibles, mun-

38 Wolfzun, Nora, "La presencia de lo excluido: algunos insumos luhmannianos para la crítica jurídica”, Filosofía del Derecho, núm. 1, 23 de septiembre de 2012.

PROBLEMA

Anuario de Filosofia y Teoría del Derecho, Núm. 8, enero-diciembre de 2014, pp. 95-124 
Esta revista forma parte del acervo de la Biblioteca Jurídica Virtual del Instituto de Investigaciones Jurídicas de la UNAM www.juridicas.unam.mx

http://biblio.juridicas.unam.mx

TEORÍA DE LOS SISTEMAS DE NIKLAS LUHMANN

dos constructores y no pasivos deudores de sus particulares regímenes de verdad. El abandono de un pensamiento sustancialista y estático abre un abanico de ideas útiles para analizar, por un lado, la existencia de una realidad social circular y dinámica, y por el otro, el protagonismo y capacidad transformadores del sistema jurídico (Cárcova, 1996: 182; Cárcova 1998: 162; Cárcova, 2007: 42; Foucault, 1995: 32; Foucault, 1996: 38; Ruiz: 2001: 30)(Wolfzun, 2012)".

Wolfzun marca como organizador conceptual que en contraste con las filosofias de la conciencia y la lógica costo-resultado de sujetos racionales, la mirada luhmanniana pondera la comunicación como elemento nodal del sistema, por considerar que sólo la comunicación es necesariamente social:

Su abordaje encuentra un punto de referencia externo, el mundo, que trasciende el sistema y representa la unidad de sentido de la diferencia entre sistema y ambiente. (En realidad, creo que no deja de ser éste un momento de incondicionalidad, un punto de vista arquimediano, que le permite problematizar la formación y la función de sistemas). Entre el mundo y el hombre, el sistema se despliega en respuesta a problemas que exigen solución. Dicho de otra manera, la evolución de los sistemas sociales son respuesta a dos problemas de referencia: la complejidad (o exceso de posibilidades en el mundo) y la contingencia (imprevisibilidad y variabilidad de dichas posibilidades). Según Piscitelli (1993: 133), nuevos principios epistemológicos permiten llevar a cabo esta torsión teórica, a saber: un principio de complejidad física, que concibe las relaciones dialógicas entre orden, desorden y organización; un principio de complejidad organizada para comprender nociones como la emergencia, la retroacción, la recursión; y un principio de complejidad lógica que concibe el vínculo entre autonomía y dependencia. (La extrapolación de categorías analíticas y esquemas conceptuales de muy diversas ramas del saber que lleva a cabo 
Esta revista forma parte del acervo de la Biblioteca Jurídica Virtual del Instituto de Investigaciones Jurídicas de la UNAM www.juridicas.unam.mx

http://biblio.juridicas.unam.mx

JORGE E. DOUGLAS PRICE

la teoria autopoiética, le otorga fecundidad en beneficio de su explicación y comprensión). ${ }^{39}$

Finaliza Wolfzun, diciendo que la inflexión luhmanniana en torno al poder nos habilita a pensar en una idea de la política entendida como conflicto, disenso, contradicción, que autores diversos:

...como Alain Badiou, Jacques Rancière, Ernesto Laclau, Jacques Derrida, entre otros, orientan sus reflexiones hacia el logro de un consenso conflictual proveedor de un espacio simbólico común para perspectivas legítimas que confrontan democráticamente (Mouffe, 2007: 58). Desde un marco conceptual que distingue entre antagonismo (relaciones entre enemigos) y agonismo (relaciones entre adversarios), la lógica agonista nosotros-ellos analiza el pluralismo no como factum sino como instancia problemática referida a prácticas específicas y a contextos particulares (Mouffe, 2007: 129). Lo que este enfoque pondera (sin pretender abarcar todas las diferencias y superar todas las formas de exclusión), es la lógica de concebir las exclusiones en términos politicos y no morales. El trazado de las fronteras entre el adentro y el afuera, lo legítimo y lo ilegítimo, constituye una decisión de índole politica y por lo tanto, siempre sujeto a discusión. ${ }^{40}$

Se trata en suma de usar la paradoja, para indicar un procedimiento, no para resolver sino para mostrar, como dice Raffaele de Giorgi, que no se ve lo que no se ve, aún si sabemos que el no ver es una condición del ver; así como olvidar es una función de la memoria.

BiBLIOGRAFÍA

CÁRCOVA, Carlos María, La opacidad del derecho, Madrid, Trotta, 1998.

39 Idem.

40 Idem. 
Esta revista forma parte del acervo de la Biblioteca Jurídica Virtual del Instituto de Investigaciones Jurídicas de la UNAM www.juridicas.unam.mx

http://biblio.juridicas.unam.mx

TEORÍA DE LOS SISTEMAS DE NIKLAS LUHMANN

GIORGI, Raffaele de, "Introducción", Redes de inclusión - La construcción social de la autoridad, México, Porrúa, 1998.

Giorgi, Raffaele de, Roma como memoria da evoluçao, trad. de G. L. Gonçalves, San Pablo, Quartier Latin, 2006.

GIORGI, Raffaele de, Jurisprudencia, Campeche, Corte Suprema de Justicia de México, 2008.

DOUGLAS PRICE, Jorge Eduardo, "Democracia, poder local y globalización. ¿Es posible constituir poderes locales autónomos?", en varios, Redes de inclusión. La construcción social de la autoridad, México, Porrúa, 1998.

DOUGlas PRICE, Jorge, Eduardo, La decisión judicial, Santa Fe, Rubinzal Culzoni, 2012.

GRÜN, Ernesto, Una visión sistémica y cibernética del derecho, Buenos Aires, Abeledo-Perrot, 1995.

GRÜN, Ernesto, Una Visión sistémica y cibernética del derecho en el mundo globalizado del siglo XXI, Buenos Aires, Dunken, 2004.

GRÜN, Ernesto, Una visión sistémica y cibernética del derecho en el mundo globalizado del Siglo XXI, Buenos Aires, LexisNexis, 2006.

LuHMANN, Niklas, Mustración sociológica y otros ensayos, Buenos Aires, SUR, 1973.

LUHMANN, Niklas, Sociología del riesgo, Guadalajara, Universidad de Guadalajara-Universidad Iberoamericana, 1992.

LUHMANN, Niklas y GIORGI, Raffaele de, Teoría de la sociedad, Guadalajara: Universidad de Guadalajara-Universidad Iberoamericana, 1993.

Russo, Eduardo Ángel, Teoría general del derecho. En la modernidad y en la posmodernidad, Buenos Aires, Abeledo-Perrot, 1995. 
Esta revista forma parte del acervo de la Biblioteca Jurídica Virtual del Instituto de Investigaciones Jurídicas de la UNAM www.juridicas.unam.mx

http://biblio.juridicas.unam.mx

JORGE E. DOUGLAS PRICE

TEUBner, Gunther, O Direito como Sistema Autopoiético, Lisboa, Fundação Calouste Gulbenkian, 1989.

Von BertalanfFy, Ludwig, Teoría General de los Sistemas, México, FCE, 1984.

WOLFZUN, Nora, "La presencia de lo excluido: algunos insumos luhmannianos para la crítica jurídica", Filosofía del Derecho, núm. 1, 23 de septiembre de 2012.

http://www.filosofiayderecho.com/rftd. 